\title{
Psychische Traumatisierung nach Arbeitsunfällen im öffentlichen Personennahverkehr. Eine Pilotstudie zu Betreuungskonzepten, Einflussfaktoren und arbeits- medizinischer Versorgung
}

\section{Psychotrauma after Occupational Accidents in Public Transportation. A Pilot Study to Support Concepts, Influencing Factors and Occupational Health-care}

\author{
Autoren \\ A. Clarner ${ }^{1}$, J. Krahl ${ }^{2}$, W. Uter ${ }^{3}$, H. Drexler ${ }^{4}$, A. Martin \\ Institute \\ Die Institutsangaben sind am Ende des Beitrags gelistet
}

Schlüsselwörter
PTSD
Psychotrauma
Erstbetreuung
Peer
Verkehr
Versorgung
Key words
pTSD
penchotrauma
peer support
public transport
health services research

Bibliografie

DOI http://dx.doi.org/

10.1055/s-0034-1395566

Gesundheitswesen 2015;

77: 225-231

(c) Georg Thieme Verlag KG

Stuttgart · New York

ISSN 0941-3790

Korrespondenzadresse

Annika Clarner, M. A.

Institut und Poliklinik

für Arbeits-, Sozial- und

Umweltmedizin

Friedrich-Alexander-Universität

Erlangen-Nürnberg (FAU)

Schillerstraße 29

91058 Erlangen

Annika.Clarner@ipasum.med.

uni-erlangen.de

\section{License terms}

\section{Zusammenfassung}

$\nabla$

Hintergrund und Zielsetzung der Studie: Unfälle, Überfälle und Suizide sind im öffentlichen Personenverkehr keine Ausnahme. In Folge von solchen Extremereignissen am Arbeitsplatz sind Fahrdienstmitarbeiter für psychische Traumatisierung besonders gefährdet. Daher sehen Unfallversicherungsträger eine Versorgung vom Unfallort bis zur betrieblichen Wiedereingliederung unter Einbezug der Arbeitsmedizin vor. Die vorliegende Studie untersucht Inzidenz, Einflussfaktoren auf Psychotraumata sowie die arbeitsmedizinische Versorgung nach Schadensereignissen.

Methodik: In einem bayrischen Verkehrsunternehmen wurden mittels Vollerhebung spezifischer Erstbetreuungseinsätze und anschließender arbeitsmedizinischer Vorstellung 59 Mitarbeiter hinsichtlich Traumafolgestörungen (TF) nach ICD-10 evaluiert.

Ergebnisse: Die Inzidenz von TF lag bei $44,1 \%$, wobei $8,5 \%$ eine posttraumatische Belastungsstörung (PTSD) als Unfallfolge des Schadensereignisses aufwiesen. Als signifikante Einflussfaktoren wurden die Art des Schadensereignisses, die Verletzungsschwere Dritter sowie eigene physische Verletzungen ermittelt. Die arbeitsmedizinische Betreuung richtete sich nach der Schwere der Erkrankung. Ausfallzeiten waren bei Mitarbeitern mit PTSD am ausgeprägtesten. Schlussfolgerung: Für das Kollektiv zeigten sich hohe Belastungsraten innerhalb des ersten Monats. Hinsichtlich der arbeitsmedizinischen Versorgung sollte besonders Augenmerk auf Fahrer gelegt werden, die bei schweren Unfällen beteiligt waren oder die selbst Verletzungen erlitten. Als Grundlage evidenzbasierter Versorgung müssen Maßnahmen nach Psychotrauma im Verkehrswesen wissenschaftlich evaluiert und gleichzeitig die ermittelten Risikofaktoren fundiert werden.

\section{Abstract \\ $\nabla$}

Background and Aim of the Study: Accidents, assaults and suicides occur quite frequently in public transportation. As a result of such extreme events at work, drivers are particularly prone to psychotrauma (PT). Therefore accident insurers stipulate support from the accident site to workplace reintegration with the inclusion of occupational medicine. The present study investigates the incidence, factors influencing psychological trauma as well as the occupational health-care after critical incidents.

Methods: In a Bavarian public transportation corporation 59 employees were evaluated according to ICD-10 for trauma-related disorders, using full collection after application of a specific mental first aid programme and a subsequent occupational health examination.

Results: The incidence of PT was $44.1 \%$, with $8.5 \%$ showing a post-traumatic stress disorder (PTSD) as consequence of the accident. As significant influencing factors the nature of the accident, the severity of third party injury, and own physical injuries were identified. The occupational medical care depended on the severity of the disease. PT led to the need for rehabilitation. Sick leave was most pronounced in drivers with PTSD.

Conclusion: The study group showed high levels of emotional strain within the first month. With respect to occupational health-care, particular attention should be paid to drivers after serious accidents or those who sustained own injuries. As foundation for evidence based health-care measures after PT in transport must be scientifically evaluated and the identified risk factors must be well-founded as well. 


\section{Einleitung

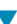

Jedes Jahr nehmen sich etwa 900 Personen mithilfe von Schienenfahrzeugen das Leben [1]. Nach dem Suizid Robert Enkes verdoppelte sich die Zahl der Nachahmungen in der Folgezeit [2]. Für Fahrdienstmitarbeiter des öffentlichen Personennahverkehrs (ÖPNV) stellen extreme Schadensereignisse wie Unfälle oder Suizide keine Seltenheit dar und bilden einen Teilbereich von belastenden Ereignissen neben Zusammenstößen mit Fahrzeugen oder Übergriffen auf das Fahrpersonal. Etwa 17\% aller Arbeitsunfälle der Branche ÖPNV/Bahnen sind nach Angaben der zuständigen Berufsgenossenschaft VBG Unfälle mitpsychischen Folgeschäden [3]. Seitens der Deutschen Gesetzliche Unfallversicherung (DGUV) besteht ein branchenübergreifendes Modellverfahren [4]. Für den Bereich des öffentlichen Verkehrswesens wurden Empfehlungen bereits Mitte der 90er Jahre entworfen [5]. Das Konzept sieht zur Reduzierung von psychischen Unfallfolgen ein mehrphasiges System, bestehend aus betrieblicher Prävention, unmittelbarer Betreuung am Unfallort, anschließender professioneller Folgebetreuung sowie betrieblicher Nachsorge vor. Ein wesentlicher Bestandteil dieses Versorgungskonzepts ist die Betreuung von potenziell Traumatisierten durch Laienhelfer (Erstbetreuer) unmittelbar am Unfallort [4,6]. Die Hauptaufgabe besteht in einer niederschwelligen, psychosozialen Versorgung des Betroffenen („sich kümmern“). Dazu gehört emotionalen Beistand leisten, abschirmen, Information über Verfahrenswege geben und an das soziale Umfeld oder professionelle Stellen (Arbeitsmedizin) vermitteln [7].

In der Praxis haben sich im Wesentlichen 3 Systeme herausgebildet: innerbetriebliche kollegiale (durch geschulte Kollegen), hierarchische (durch übergeordnete Mitarbeiter, u.a. Verkehrsmeister) oder externe Erstbetreuung durch beauftragte Dienstleister. Die Überlegenheit einzelner Systeme ist unklar. Ein weiterer Bestandteil des Betreuungsverfahrens ist die anschließende arbeitsmedizinische Versorgung. Hierbei können Arbeitsmediziner mit der Steuerung des Heilverfahrens beauftragt und zu Beginn 5 probatorische psychotherapeutische Sitzungen bei der zuständigen BG beantragen $[4,6]$. $\mathrm{Zu}$ weiteren betriebsärztlichen Aufgaben siehe [7].

Obwohl viele Verkehrsunternehmen in Deutschland über ein Erstbetreuersystem verfügen, ist wenig über Inzidenz von, und Einflussfaktoren auf, Psychotraumata in der Regelversorgung erstbetreuter Fahrdienstmitarbeiter bekannt. Der bisherige Erkenntnisstand stützt sich überwiegend auf Expertenmeinungen, weshalb international Studien zur Wirksamkeit gefordert werden [8].

Bisherige Untersuchungen im öffentlichen Personenverkehr fanden überwiegend an Eisenbahnlok- oder U-Bahnführern nach sogenannten „person under train“ (PUT) Ereignissen statt. Darunter werden Schadensereignisse verstanden, in denen eine Person absichtlich oder unbeabsichtigt mit einem Schienenfahrzeug zusammenstößt und infolge schwerverletzt oder getötet wird [9-11].

Die Prävalenz von Traumafolgeerkrankungen (TF) nach PUT variiert stark. Am Häufigsten wurde das Auftreten posttraumatischer Belastungsstörungen (engl. Post-traumatic stress disorder, PTSD) untersucht, welche zwischen 4-18\% [11-14], lagen.

Teilweise war auch subsyndromale PTSD (18-50\%) [14-16] Gegenstand der Forschung. Andere TF wurden seltener untersucht $[11,13]$. Einige Studien untersuchten die Häufigkeit akuter Belastungsstörungen (ASD) (4-25\%) [11,15] oder das Auftreten psychophysiologischer Reaktionen (33\%) [17]. Die Vergleichbar- keit der Ergebnisse wird insbesondere durch unterschiedliche methodische Ansätze erschwert.

Als individuelle Einflussfaktoren auf die Entstehung von PTSD erhöhte sich das Risiko für Fahrer nach PUT bei jüngerem Alter, und die Prognose verschlechterte sich bei Schuldgefühlen, Angst und Entfremdung [18, 19].

Akuter, dauerhafter oder zusätzlicher Stress stellte einen Risikofaktor für psychische Beeinträchtigung dar [12]. Untersuchungen an Unfallopfern wiesen einen Zusammenhang zur Stärke eigener physischer Verletzung auf [20] welcher bislang jedoch nicht an verunglückten Fahrern selbst untersucht wurde. Hinsichtlich arbeitsplatz- und ereignisbezogener Faktoren wurden übereinstimmend die PUT-Ereignisse selbst für die Entstehung psychischer Folgeerkrankungen identifiziert. Dabei erhöhten frühere PUT zusätzlich das Risiko [9,21]. Obwohl die Verletzungsschwere des Opfers oder der verursachenden Person als Einflussfaktor gilt, wurde sie selbst bislang kaum untersucht [9]. Gleiches gilt auch für die Art der Schadensereignisse. Näheres siehe [22].

Zusammenfassend fehlen für den öffentlichen Personennahverkehr in Deutschland Untersuchungen an Fahrerkollektiven nach PUT und anderen Extremereignissen. Die in der Regelversorgung geleistete Erstbetreuung ist bislang nicht erforscht und Traumafolgeerkrankungen sowie Einflussfaktoren nach Schadensereignissen bei Mitarbeitern aus dem Fahrdienst sind weitgehend unbekannt.

Zielsetzung: Die vorliegende Studie untersucht erstmalig ein Gesamtkollektiv von erstbetreuten Fahrdienstmitarbeiter nach $\mathrm{Ar}$ beitsunfällen im ÖPNV. Dabei soll in Anlehnung an [23]:

a) die Häufigkeit von Traumafolgeerkrankungen, v.a. von PTSD, bei erstbetreuten Fahrdienstmitarbeitern untersucht werden,

b) festgestellt werden, welchen Einfluss individuelle, arbeitsplatz-, ereignisbezogene Faktoren hinsichtlich akuter TF haben sowie

c) erhoben werden, welche Folgen hinsichtlich Ausfallzeiten und arbeitsmedizinischer Versorgung nach Schadensereignissen resultieren.

\section{Material und Methoden}

$\nabla$

\section{Stichprobe}

Als Verkehrsbetrieb konnte die VAG Verkehrs-Aktiengesellschaft Nürnberg gewonnen werden. Die Datengrundlage bildete die Seitens des Unternehmens geführte Einsatzstatistik entsandter Erstbetreuer. Es handelte sich um eine Vollerhebung von 73 Erstbetreuereinsätzen zwischen Einführung des Systems im Juli 2004 und Dezember 2009. Von allen 73 Einsätzen konnten 72 Erstbetreute ermittelt werden. In der nachfolgenden arbeitsmedizinischen Untersuchung wurden 66 Mitarbeiter vorstellig. Bei 2 Mitarbeitern fehlte der betriebsärztlicher Bericht, bei 4 weiteren waren die psychiatrischen Befunde nicht vorhanden. Insgesamt lagen bei 60 Mitarbeitern (91\% der Vorstelligen) alle notwendigen Befunde vor. Eine Person wurde aufgrund der Zugehörigkeit zu den Servicediensten von der Untersuchung ausgeschlossen ( $\bullet$ Abb. 1 ).

Somit lag bei 59 Personen des Fahrdiensts eine vollständige Dokumentationsbasis vor. 55 (93\%) waren männlich und 4 (7\%) weiblich. Im Mittel betrug das Alter $41(\mathrm{SD}=7,5)$, das Dienstalter 10 Jahre $(S D=6,8)$. 


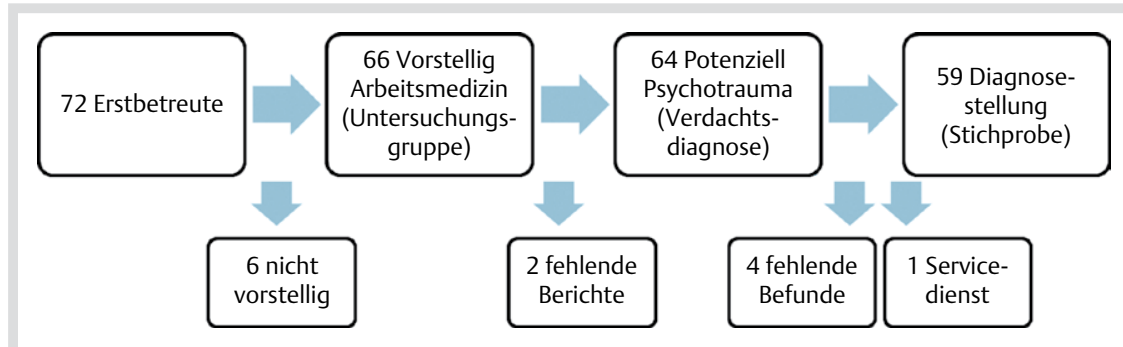

Abb. 1 Flussdiagramm des Einschlusses von Teilnehmern in die Studie.

\section{Design}

Das Untersuchungsdesign entspricht einer Kohortenstudie mit zurückverlagertem Ausgangspunkt (historisch). Die Datenerfassung erfolgte anhand einer eigens entwickelten Rohdatenmatrix unter Einbezug von Sozialberatung, Arbeitssicherheit und Umweltschutz, Unfallabteilung, Personalabteilung und Arbeitsmedizin.

Zur Wahrung der Interessen und zum Schutz der Mitarbeiter waren Betriebsrat und Datenschutzbeauftragter während des gesamten Studienverlaufs involviert, einschließlich der Abstimmung aller berücksichtigungsfähigen Variablen. Die Studie wurde juristisch geprüft und eine Forschungskooperation vereinbart. Die Studie wurde in Übereinstimmung zur Deklaration von Helsinki konzipiert und durchgeführt.

\section{Instrumente und Diagnostik}

Alle Angaben entstammen den Dokumentationssystemen des Unternehmens. Zur Messung und Operationalisierung arbeitsplatz- und ereignisbezogener Faktoren wurden Fahrzeugtyp (Bus, Straßenbahn, U-Bahn), Art des Schadensereignisses (differenziert nach Unfällen (Zusammenstoß zwischen Fahrzeug und Person), Zusammenstöße mit Fahrzeugen, suizidalen Ereignissen, Bedrohungen/Angriffe oder sonstige Ereignisse), Verletzungsschwere Dritter (orientiert am Polizeibericht, Skalierung: keine, leicht, schwer, getötet), geschätzte Schadenshöhe am Fahrzeug (kein, leichter, mittlerer, schwerer Schaden), Zeitpunkt der Arbeitseinstellung nach Arbeitsunfall (sofort vs. nein/später), vorheriges Schadensereignis (auf Basis früherer Vorstellungen eines Mitarbeiters in der Arbeitsmedizin nach vergleichbaren Unfallereignissen) sowie verzögerte arbeitsmedizinische Betreuung (definiert als Freitag bis Sonntag), wenn es nicht zu einer arbeitsmedizinischen Vorstellung bereits am selben Tag kam) erhoben.

In Kombination mit der Verletzungsschwere Dritter konnten Schadensereignisse nach PUT und anderen Ereignissen differenziert werden. Als PUT-Ereignisse wurden Vorfälle definiert, in denen (i) eine Person (ii) unbeabsichtigt oder beabsichtigt (iii) im direkten Kontakt mit einem Fahrzeug kam und (iv) hierdurch verletzt wurde. Für die Analyse wurden PUT in 2 Versionen spezifiziert. In PUT(V) sind Ereignisse mit Personen zusammengefasst worden, in denen es unabhängig von der Schwere zu einer Verletzung der beteiligten Person kam. Für PUT(ST) wurden nur Ereignisse eingeschlossen, bei denen es zu schweren oder tödlichen physischen Schadensfolgen kam.

Als individuelle Faktoren wurde das Alter und die Dauer der Betriebszugehörigkeit des Mitarbeiters zum Unfallzeitpunkt in Jahren sowie physische Schadensfolgen (ja/nein) auf Basis der dokumentierten Vorfallmeldungen gemessen. Zur Messung von Ereignisfolgen wurden Wiedereingliederungsmaßnahmen vor regulärer Aufnahme des Fahrdienstes (ja/nein), die Anzahl der Termine in der Arbeitsmedizin nach Unfallereignis, ergänzend der Zeitpunkt der Vorstellung in Tagen nach einem Ereignis sowie die unmittelbaren Ausfallzeiten nach einem Schadensereignis bis zur Wiederaufnahme der Arbeitstätigkeit ermittelt.

\section{Diagnostik der Traumafolgestörungen (TF)}

Die Erfassung von TF erfolgte auf Grundlage der arbeitsmedizinischen sowie neurologisch/psychiatrischen Befunde. Dabei waren betroffene Mitarbeiter in berufsgenossenschaftlich anerkannten psychiatrischen Praxen vorstellig. Im ersten Schritt wurden die seitens der Arbeitsmedizin gestellten Verdachtsdiagnosen und die damit verbundenen Überweisungen zum Facharzt erhoben. Mitarbeiter ohne auffällige Symptomatik wurden nicht überwiesen. Im Überweisungsbericht wurden ggf. frühere berufliche Traumatisierungen angegeben. Die seitens der Fachpraxis vergebene Klassifikation nach ICD-10 (TF) wurde zur Auswertung übernommen. Überdies wurden die ermittelten TF hinsichtlich lang- und kurzfristiger Symptomatik spezifiziert (keine, kurz-, langfristig). Hierbei bezogen sich kurzfristige Erkrankungen auf die ersten 4 Wochen nach einem Schadensereignis. Bei allen langfristigen psychischen Störungen handelte es sich um das Vollbild klassifizierter PTSD (ICD-10F 43.1) mit einer Dauer von mehr als einem Monat.

\section{Statistische Analyse}

Die statistische Auswertung erfolgte mittels SPSS 22 sowie R (Version 3.1.0). Die Analysen erfolgten bivariat. Als Testverfahren diente der exakte Test nach Fisher (nominal/ordinale Variablen). Daneben wurde der Kendall-tau-b-Koeffizient (ordinal/ ordinale Variablen) sowie für metrisch/ordinal-skalierte Variablen bei Normalverteilung eine einfaktorielle Varianzanalyse oder alternativ der Kruskal-Wallis-Test verwendet. Die Testung erfolgte 2-seitig zu einem Signifikanzniveau von $\mathrm{p}<0,05$; angesichts der explorativen Natur ohne alpha-Adjustierung.

\section{Ergebnisse}

\section{Häufigkeit psychischer Traumatisierung und akuter Traumafolgestörungen}

Nach einem Schadensereignis mit anschließender Erstbetreuung wurden von seiten der Arbeitsmedizin aufgrund akuter psychischer Traumafolgesymptomatik 47\% $(n=28)$ der Betroffenen in eine psychiatrische Praxis überwiesen ( $\bullet$ Tab. 1). In $35 \%$ $(n=20)$ aller Fälle wurde ein erstmaliges Psychotrauma zur weiteren Abklärung festgestellt. Weitere $12 \%(n=7)$ erlebten bereits die zweite Traumatisierung innerhalb ihrer Fahrdiensttätigkeit. Eine Gruppe von 5\% $(n=3)$ zeigte aufgrund des aktuellen Schadensereignisses keine psychische Verdachtssymptomatik, diese war jedoch im Rahmen eines früheren beruflichen Ereignisses arbeitsmedizinisch dokumentiert worden. Die arbeitsmedizinisch gestellte Diagnose wurde bis auf 2 Fälle durch die psychia- 
Tab. 1 Deskription psychischer Traumatisierung im Kollektiv $(n=59)$.

\begin{tabular}{lll}
$\begin{array}{l}\text { Psychische Traumatisierung } \\
\text { Überweisung zum Facharzt }\end{array}$ & [n] & $\%$ \\
\hline $\begin{array}{l}\text { nein } \\
\text { ja }\end{array}$ & 31 & 53 \\
$\begin{array}{l}\text { Verdacht Psychotrauma } \\
\text { keines }\end{array}$ & 28 & 47 \\
\hline nur vergangenes & 28 & 48 \\
\hline erstes Psychotrauma & 3 & 5 \\
\hline $\begin{array}{l}\text { 2 oder mehrere Psychotrauma } \\
\text { Traumafolgestörungen * }\end{array}$ & 20 & 35 \\
\hline $\begin{array}{l}\text { akute Belastungsreaktion (F43.0) } \\
\text { Anpassungsstörung (F43.2) }\end{array}$ & 7 & 12 \\
\hline posttraumatische Belastungsstörung (F43.1) & 26 & 44,1 \\
* Gesamt ohne Mehrfachnennungen & 16 & 27 \\
& 9 & 15 \\
\hline & 5 & 8,5 \\
\hline
\end{tabular}

trischen Befunde bestätigt. Die Inzidenz gesicherter akuter TF betrug somit insgesamt $44,1 \%(n=26)$. Davon entwickelten $8,5 \%$ $(\mathrm{n}=5)$ langfristige TF.

\section{Potenzielle Einflussfaktoren und aufgetretene Folgen}

Hinsichtlich arbeitsplatz- und ereignisbezogener Einflussfaktoren standen Schadensereignisse mit schienengebundenen Fahrzeugen (Straßenbahn, U-Bahn) (83\%) im Vordergrund ( $\bullet$ Tab. 2). Dabei kam es am häufigsten zu Unfällen (51\%). Die Unterteilung nach PUT-Ereignissen belief sich im Falle von PUT(V) einschließlich Leichtverletzter auf $63 \%$ der Vorfälle, wobei $46 \%$ aller Ereignisse PUTs im Sinne PUT(ST) mit Schwerverletzten oder Getöteten waren.

Für beteiligte Passanten oder Passagiere endete fast die Hälfte aller Vorfälle mit schwerer Verletzung oder Tod. Der am Fahrzeug geschätzte Schaden war zu $41 \%$ leicht bis mittel. Entgegen der üblichen Praxis im Unternehmen stellten $8 \%$ der Betreuten ihre Diensttätigkeit nicht oder erst später ein. Über ein Drittel der Untersuchten hatte bereits früher ähnliche Ereignisse erlebt. Eine verzögerte Betreuung durch die Arbeitsmedizin aufgrund der Verteilung der Wochentage betraf 20\% der Mitarbeiter. Körperliche Verletzungen von Mitarbeitern wurden selten beobachtet (16\%). Hierbei handelte es sich ausschließlich um leichte Verletzungen, am häufigsten um ein Halswirbelsäulen-Schleudertrauma.

Die arbeitsmedizinische Betreuung erfolgte zeitnah (Median (M) in Tagen =1,00, Q1-Q3 =0,0-1,0). Vor Wiederaufnahme ihrer regulären Fahrdiensttätigkeit erhielten $42 \%$ der Mitarbeiter eine Form von Wiedereingliederungsmaßnahmen. Diese reichten von begleiteten Fahrten bis zu Umschulungsmaßnahmen. Die unmittelbare Ausfallzeit betrug im Median 3 Tage $(M=3,00$, Q1-Q3 =4,0-16,0), wobei 70\% $(n=39)$ mindestens einen Tag ausfielen.

\section{Assoziation zwischen Prädiktoren und \\ Traumafolgestörungen}

In den bivariaten Analysen zum Einfluss von arbeitsplatz-, ereignisbezogenen sowie individuellen Faktoren auf TF wurden die Art des Schadensereignisses (PUT(ST)), die Verletzungsschwere Dritter, sowie eigene physische Verletzungen der Mitarbeiter als signifikante Faktoren identifiziert. Demnach wurden TF signifikant häufiger bei PUT mit schweren Verletzungen Dritter im Vergleich zu anderen Schadensereignissen beobachtet. Wie in $\bigcirc$ Tab. 3 dargestellt, gab es keine signifikanten Ergebnisse bezüglich anderer erfasster Faktoren.
Tab. 2 Deskription von Einflussfaktoren sowie Folgen und Versorgung.

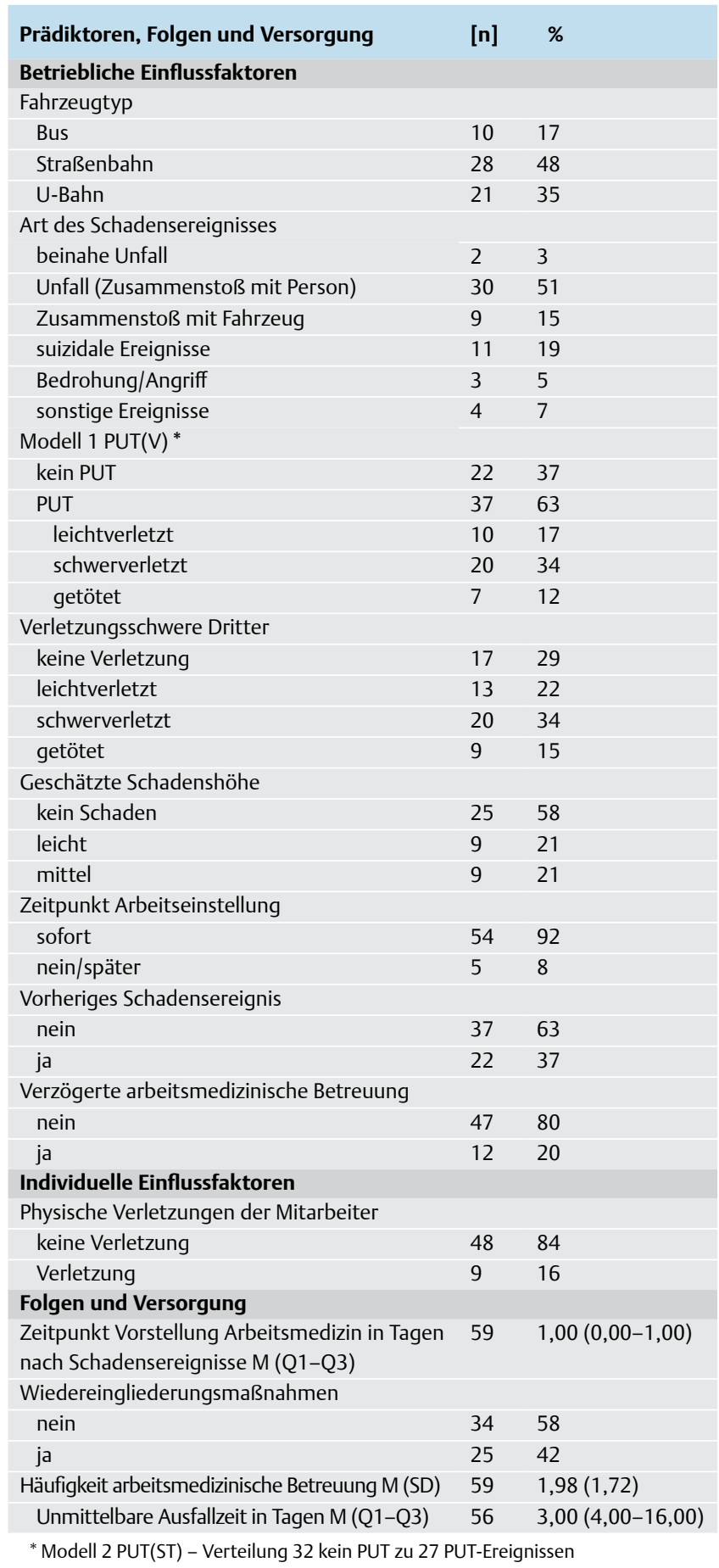

Konsequenzen von Traumafolgestörungen

Hinsichtlich der erfassten Folgen und arbeitsmedizinischen Versorgung ergaben sich signifikante Zusammenhänge mit entstandenen TF nach einem Schadensereignis ( $\bullet$ Tab. 3). Nicht nur Wiedereingliederungsmaßnahmen, auch die Intensität der arbeitsmedizinischen Betreuung war assoziiert mit der Art und Dauer der entwickelten TF. Die für arbeitsmedizinische Betreuung (Kendall-tau-b=0,517) und Ausfallzeit (Kendall-tau-b= 0,506) berechneten Korrelationen lagen im mittleren Bereich. Nicht zuletzt gingen TF mit erheblichen Ausfallzeiten der Mitarbeiter einher. So betrugen die Ausfallzeiten bei langfristigen Erkrankungen im Median 41,00 Tage (Q1-Q3=21,0-54,5) im Ver- 
Tab. 3 Bivariate Analyse zu Prädiktoren, Folgen und Versorgung von Traumafolgestörungen.

\begin{tabular}{|c|c|c|c|c|c|c|}
\hline \multirow[t]{2}{*}{ Prädiktoren und Folgen } & \multicolumn{6}{|c|}{ Traumafolgestörungen [\%] } \\
\hline & & [n] & Keine & Kurzfristige & Langfristige & Analyse \\
\hline \multicolumn{7}{|l|}{ Betriebliche Einflussfaktoren } \\
\hline \multicolumn{7}{|l|}{ Fahrzeugtyp } \\
\hline Bus & & 10 & 50 & 50 & 0 & $\mathrm{p}=0,711^{\mathrm{a}}$ \\
\hline Straßenbahn & & 28 & 61 & 32 & 7 & \\
\hline U-Bahn & & 21 & 53 & 33 & 14 & \\
\hline \multicolumn{7}{|l|}{ Art des Schadensereignisses } \\
\hline \multicolumn{7}{|l|}{ PUT(V) } \\
\hline kein PUT & & 22 & 68 & 32 & 0 & $\mathrm{p}=0,153^{\mathrm{a}}$ \\
\hline PUT (leicht-schwer-getötet) & & 37 & 49 & 38 & 13 & \\
\hline \multicolumn{7}{|l|}{ PUT(ST) } \\
\hline kein PUT & & 32 & 69 & 31 & 0 & $p=0,001^{a}$ \\
\hline PUT (schwer-getötet) & & 27 & 41 & 41 & 18 & \\
\hline \multicolumn{7}{|l|}{ Verletzungsschwere Dritter } \\
\hline keine Verletzung & & 17 & 65 & 35 & 0 & $p=0,024^{b}$ \\
\hline leichtverletzt & & 13 & 77 & 23 & 0 & \\
\hline schwerverletzt & & 20 & 45 & 40 & 15 & \\
\hline getötet & & 9 & 33 & 45 & 22 & \\
\hline \multicolumn{7}{|l|}{ Geschätzte Schadenshöhe } \\
\hline kein Schaden & & 25 & 56 & 40 & 4 & $p=0,100^{a}$ \\
\hline leicht & & 9 & 33 & 33 & 33 & \\
\hline mittel & & 9 & 78 & 22 & 0 & \\
\hline \multicolumn{7}{|l|}{ Zeitpunkt Arbeitseinstellung } \\
\hline sofort & & 54 & 60 & 31 & 9 & $p=0,102^{a}$ \\
\hline nein/später & & 5 & 20 & 80 & 0 & \\
\hline \multicolumn{7}{|l|}{ Vorheriges Schadensereignis } \\
\hline nein & & 37 & 54 & 35 & 11 & $\mathrm{p}=0,845^{\mathrm{a}}$ \\
\hline ja & & 22 & 59 & 36 & 4 & \\
\hline \multicolumn{7}{|l|}{ Verzögerte Betreuung } \\
\hline nein & & 47 & 55 & 36 & 9 & $p=1^{a}$ \\
\hline ja & & 12 & 58 & 33 & 8 & \\
\hline Individuelle Einflussfaktoren & & [n] & Keine & Kurzfristige & Langfristige & Analyse \\
\hline \multirow[t]{2}{*}{ Alter } & MW & 59 & 40,91 & 41,86 & 42,60 & \\
\hline & SD & & 7,49 & 6,04 & 11,13 & $p=0,872^{c}$ \\
\hline \multirow[t]{2}{*}{ Betriebszugehörigkeitsdauer } & M & 58 & 7,00 & 13,00 & 5,00 & \\
\hline & (Q1-Q3) & & $4,0-17,0$ & $4,5-13,0$ & $2,0-11,5$ & $p=0,425^{d}$ \\
\hline \multicolumn{7}{|l|}{ Physische Verletzungen der Mitarbeiter } \\
\hline keine Verletzung & & 48 & 54 & 42 & 4 & $\mathrm{p}=0,020^{\mathrm{a}}$ \\
\hline Verletzung & & 9 & 56 & 11 & 33 & \\
\hline Folgen und Versorgung & & {$[\mathrm{n}]$} & Keine & Kurzfristige & Langfristige & Analyse \\
\hline \multicolumn{7}{|l|}{ Wiedereingliederungsmaßnahmen } \\
\hline nein & & 34 & 74 & 23 & 3 & $\mathrm{p}=0,026^{\mathrm{a}}$ \\
\hline ja & & 25 & 32 & 52 & 16 & \\
\hline Häufigkeit arbeitsmedizinische & MW & 59 & 1,35 & 2,10 & 5,60 & $\mathrm{p}<0,001^{\mathrm{b}}$ \\
\hline Betreuung & SD & & 0,73 & 0,94 & 3,85 & 0,517 \\
\hline \multirow{2}{*}{ Unmittelbare Ausfallzeit in Tagen M } & MW & 56 & 1,00 & 4,50 & 41,00 & $\mathrm{p}<0,001^{b}$ \\
\hline & (Q1-Q3) & & $0,00-3,00$ & $1,25-9,75$ & $21,0-54,5$ & 0,506 \\
\hline
\end{tabular}

a berechnet mittels Exakter Test nach Fischer; ${ }^{\mathrm{b}}$ Kendall-Tau-b; ' Einfaktorielle Varianzanalyse; ${ }^{\mathrm{d}}$ Kruskal-Wallis-Test

gleich zu kurzfristigen ( $M=4,50, \mathrm{Q} 1-\mathrm{Q} 3=1,25-9,75)$ und keinen $(\mathrm{M}=1,00, \mathrm{Q} 1-\mathrm{Q} 3=0,00-3,00)$. Zusammenfassend zeigte sich, dass TF mit erheblichen Ausfallzeiten für PTSD und erhöhter arbeitsmedizinischer Nachsorge verbunden waren.

\section{Diskussion}

Psychische Belastungen und TF einschließlich der Unfallversicherungsträger empfohlenen Erstbetreuung sind bislang nur unzureichend in Verkehrsunternehmen des ÖPNV erforscht. Zielsetzung der vorliegenden Studie war die Untersuchung psychi- scher Traumatisierung nach Arbeitsunfällen im ÖPNV. Hierbei wurde erstmalig ein Gesamtkollektiv erstbetreuter Fahrdienstmitarbeiter hinsichtlich Häufigkeit akuter TF, Einflussfaktoren einschließlich Folgen und arbeitsmedizinsicher Versorgung analysiert.

Nach Schadensereignissen zeigten insgesamt $44,1 \%$ der erstbetreuten Mitarbeiter eine TF; davon 35,6\% (Akute Belastungsreaktion oder Anpassungsstörung), 8,5\% der Fahrdienstmitarbeiter entwickelten eine PTSD. Ein Vergleich mit früheren Studien fällt aufgrund unterschiedlicher Methodik schwer. In Bezug auf TF (ASD) war die Inzidenz höher als in der Studie von Myrtek et al. (25\%) [15]. Bezogen auf Messungen unmittelbar nach PUT 
(11\% nach DSM-IV diagnostizierten Erkrankungen) [12], lag die unsererseits ermittelte Inzidenz mehr als 3-mal so hoch. Innerhalb der ersten Stunden wurden bei einem Drittel psycho-physiologische Reaktionen [17], 15 Tage später 17,9\% mit psychischen Leiden und Beeinträchtigungen, neben einer äußerst geringen Anzahl an akuten Belastungsstörungen (4\%) und Major-Depressionen (1,5\%). ermittelt [11]. Die in unserem Kollektiv festgestellten Raten fielen also insgesamt höher aus als in den bisherigen Erhebungen. Ein Grund könnte im Messzeitpunkt selbst innerhalb der ersten Tage nach einem Schadensereignis und dem Einschluss diagnostizierter akuter Belastungsreaktionen liegen. Andererseits könnten frühere Ergebnisse teilweise aufgrund des „Recall-bias“ verzerrt sein $[15,17]$. Im Vergleich zu ermittelter PTSD (nach einem Monat) in früheren Untersuchungen $(15,1-17,8 \%)[13,14,16]$ fand sich in der vorliegenden Studie eine deutlich geringere Inzidenz. Einerseits könnte dies auf unterschiedliche Methodik oder fehlende Versorgungsstrukturen zurückzuführen sein. Andererseits wurde PTSD in dieser Studie nach einem breiteren Spektrum an Schadensereignissen und nicht nur auf reine PUT-Ereignisse bezogen untersucht. Dennoch fanden auch Myrtek et al. 8\% PTSD bei Eisenbahnführern [15].

Insgesamt wiesen die untersuchten Fahrdienstmitarbeiter eine höhere Inzidenz kurzfristiger TF im Gegensatz zu bislang untersuchten Zug- und U-Bahnführern auf, während PTSD seltener waren.

Als Einflussfaktoren auf Psychotraumata wurden im vorliegenden Kollektiv die Art des Schadensereignisses (schwere und tödliche PUTs), die Verletzungsschwere Dritter wie auch eigene physische Verletzungen von Fahrern nachgewiesen. In Abhängigkeit der zur Verletzungsschwere analysierten Schadensereignisse sprechen die Ergebnisse dafür, dass ein erhöhtes Risiko für TF neben klassischen PUT auch nach schweren Verletzungsfolgen dritter Personen (z. B. Fahrgäste) besteht. Für den ÖPNV aber auch den Zugverkehr sollte sich hierdurch eine Erweiterung des Fokus ergeben. Zusammenstöße mit Fahrzeugen insbesondere bei Gleisübergängen können für PKW-Insassen ebenfalls mit erheblichen Verletzungsfolgen einhergehen. Diese sind jedoch in der bisherigen PUT-Definition unberücksichtigt. Aus diesem Grund sollten, ähnlich wie bereits von Vastshelle et al., „on-thetrack-accidents“ anstatt reinen PUT-Ereignissen untersucht werden [10]. Das Risiko psychischer Traumatisierung war für Fahrer mit erlittener physischer Unfallfolge erhöht. Diesem Faktor könnte für den ÖPNV eine besondere Rolle zukommen. Aus Untersuchungen an Unfallopfern ist die eigene Verletzungsschwere als Risikofaktor bekannt [23]. Obwohl es sich bei den untersuchten Fahrern nur um leichte Verletzungen der HWS handelte, ist das Auftretens derartiger Verletzungen insbesondere nach Zusammenstößen mit anderen Fahrzeugen wahrscheinlich. Inwieweit sich der Faktor selbst zur Vorhersage späterer PTSD eignet, müsste weitergehend - insbesondere im Kontext von Verletzungsfolgen Dritter - multivariat überprüft werden.

Folgen und arbeitsmedizinische Versorgung bezogen sich auf den Wiedereintritt in den Fahrdienst bzw. die Ausfallzeiten nach einem Schadensereignis. Vor dem Hintergrund unterschiedlicher Sozialversicherungssysteme waren Ausfallzeiten bei Fahrdienstmitarbeitern mit PTSD-annähernd mit Londoner U-Bahn und Zugführern vergleichbar [13]. Insgesamt war die Verteilung bei mindestens einem Tag Krankschreibung überdies ähnlich wie bei französischen und deutschen Lokführern (69\%) [12,14], wohingegen sich höhere Ausfallhäufigkeiten bei schwedischen U-Bahnführern (81,4\%) fanden [9]. Verglichen mit deutschen
Triebfahrzeugführern wurden im Untersuchungskollektiv durchschnittlich geringere Ausfallzeiten ermittelt [14,15].In Hinblick auf Wiedereingliederungsmaßnahmen und arbeitsmedizinische Betreuung zeigten sich Zusammenhänge mit aufgetretenen TF. Dies unterstreicht einerseits den für Betriebe und Arbeitsmedizin entstehenden Versorgungsbedarf von Traumatisierten nach Schadensereignissen, macht aber zugleich die notwendige Kooperation und Zusammenarbeit mit Psychotherapeuten oder Psychiatern im Heilverfahren deutlich.

Diese Studie verwendete vorhandene Daten aus betrieblichen Abläufen, arbeitsmedizinischer und psychiatrischer Versorgung. Obwohl ein derartiges Vorgehen in der Versorgungsforschung keine Ausnahme darstellt [24], mussten Einschränkungen hinsichtlich fehlender Angaben zu eingesetzten Testverfahren akzeptiert werden. Angesichts durchwegs berufsgenossenschaftlich anerkannter psychiatrischen Praxen sollten jedoch die ermittelten TF, klassifiziert nach ICD-10, als belastbar angesehen werden. Alle Diagnosen wurden sozialversicherungsrechtlich anerkannt. Die subjektive Verarbeitung wie auch der Einfluss sozialer Unterstützung konnte studientechnisch nicht erfasst werden. Eine wesentliche Stärke der Untersuchung liegt in ihrem Studiendesign. Aufgrund der prospektiven Untersuchungsrichtung konnten Selektionseffekte, etwa durch eine verringerte Teilnahmequote verhindert werden. Da es sich um eine Vollerhebung eines gesamten Fahrerkollektivs handelt, waren valide Aussagen zur Versorgungsstruktur und -praxis möglich.

Anlässlich der vorliegenden Ergebnisse stellen psychische Traumatisierung nach Schadensereignissen Betriebe im ÖPNV vor erheblichen Handlungsbedarf, der sich nicht zuletzt in der Frage geeigneter arbeitsmedizinischer und psychotherapeutischer Versorgung für Betroffene mit Traumafolgeerkrankungen auswirkt. Im Rahmen evidenzbasierter Versorgungsforschung [25] ist es daher notwendig, die ermittelten Einflussfaktoren zu fundieren und Maßnahmen der Regelversorgung wissenschaftlich zu evaluieren. Dies betrifft nicht nur die Erstbetreuung im Verkehrswesen sondern den Bereich der Akutversorgung nach berufsbedingter Traumatisierung insgesamt.

\section{Danksagung}

$\nabla$

Diese Publikation entstand im Rahmen des von der Deutschen Gesetzlichen Unfallversicherung (DGUV) geförderten Forschungsprojekts DGUV-FP 335. Die Autoren danken der DGUV. Besonderer Dank geht an die Verantwortlichen und Mitarbeiter der VAG Verkehrs-Aktiengesellschaft Nürnberg für ihr großes Engagement und die geleistete personelle und finanzielle Unterstützung. Des Weiteren danken die Autoren Herrn Dipl. Psych. et. Theol. G. Jose sowie Herrn Prof. Dr. U. Sax für ihre Beratung bei der Studiendurchführung. Diese Arbeit wurde durch ein Begabtenstipendium der Hanns-Seidel-Stiftung aus Mitteln des Bundesministeriums für Bildung und Forschung (BMBF) gefördert. Der vorliegende Artikel ist kumulativer Bestandteil zur Erreichung des Grades Dr. rer biol. hum.

Interessenkonflikt: Diese Studie wurde finanziell durch die VAG Verkehrs-Aktiengesellschaft Nürnberg gefördert.

Institute

1 Institut und Poliklinik für Arbeits-, Sozial- und Umweltmedizin, FriedrichAlexander-Universität Erlangen-Nürnberg (FAU), Erlangen

${ }^{2}$ Technologietransferzentrum Automotive der Hochschule Coburg, Coburg 
${ }^{3}$ Institut für Medizininformatik, Biometrie und Epidemiologie, FriedrichAlexander-Universität Erlangen-Nürnberg (FAU), Erlangen

${ }^{4}$ Institutsleiter, Institut und Poliklinik für Arbeits-, Sozial- und

Umweltmedizin, Erlangen

${ }^{5}$ Klinische Psychologie und Psychotherapie, Bergische Universität Wuppertal, Wuppertal

\section{Literatur}

1 European Railway Agency. Hrsg. Intermediate report on the development of railway safety in the European Union. In: 2013

2 Ladwig KH, Kunrath S, Lukaschek $K$ et al. The railway suicide death of a famous German football player: impact on the subsequent frequency of railway suicide acts in Germany. J Affect Disord 2012; 136: 194-198

3 Weymann J. Prävention von Traumatisierungen bei Beschäftigten im Fahrdienst. Zeitschrift für Psychotraumatologie, Psychotherapiewissenschaft, Psychologische Medizin 2010; 8: 23-31

4 Drechsel-Schlund C, Feddern K, Klinkert $M$ et al. Reha-Management bei Traumatisierungen nach Arbeitsunfällen. Zeitschrift für Psychotraumatologie, Psychotherapiewissenschaft, Psychologische Medizin 2010; 8: 33-48

5 Deutsche Gesetzliche Unfallversicherung e.V. (DGUV). Betreuung von Schockverletzten bei der BG Bahnen. Im Internet: http://www.dguv. de/inhalt/praevention/praev_lohnt_sich/best_practice/unfallschock/ index.jsp Stand: 10.01.2013

6 VBG. Trauma und Psyche. Betreuung von Beschäftigten in Verkehrsunternehmen nach traumatischen Ereignissen In: Warnkreuz Spezial Nr. 2. (April 2012). Im Internet: http://www.vbg.de/oepnv_bahnen/bahn/ wk2/inhalt.htm Stand: 08.03.2012

7 Clarner A, Hopf $H-G$, Drexler H. Versorgung psychisch traumatisierter Mitarbeiter nach Arbeitsunfällen im öffentlichen Personennahverkehr. Bedeutung für die arbeitsmedizinische Versorgungspraxis. ASU 2014; 49: 527-530

8 Creamer MC, Varker T, Bisson J et al. Guidelines for peer support in high-risk organizations: an international consensus study using the Delphi method. J Trauma Stress 2012; 25: 134-141

9 Theorell T, Leymann H, Jodko $M$ et al. "Person under train" incidents: medical consequences for subway drivers. Psychosom Med 1992; 54 : 480-488

10 Vatshelle Å, Moen BE. Serious on-the-track accidents experienced by train drivers: Psychological reactions and long-term health effects. J Psychosom Res 1997; 42: 43-52

11 Limosin F, Loze J-Y, Cothereau C et al. A prospective study of the psychological effects of "person under train" incidents on drivers. J Psychiatr Res 2006; 40: 755-761

12 Cothereau C, de Beaurepaire C, Payan C et al. Professional and medical outcomes for French train drivers after "person under train" accidents: three year follow up study. Occup Environ Med 2004; 61: 488-494
13 Tranah T, Farmer RDT. Psychological reactions of drivers to railway suicide. Soc Sci Mede 1994; 38: 459-469

14 Pütz S. Posttraumatische Belastungsstörung bei Lokomotivführern nach (Dienst-) Unfällen [Dissertation]. Köln: Universität zu Köln, 2002; 106

15 Myrtek M, Itte H, Zimmermann W et al. Psychische Bewältigung von Unfällen bei Lokomotivführern: Die Relevanz von Copingfragebögen zur erfassung von funktionalen und dysfunktionalen Copingprozessen. Z Klin Psychol 1994; 23: 293-304

16 Denis $D$. Die Angst fährt immer mit.... Wie Lokführer traumatisierende Schienenunfälle bewältigen. Train drivers' coping with traumatizing railroad accidents. Freie Universität Berlin, Fachbereich Erziehungswissenschaft und Psychologie. Kroening: Asanger, 2004

17 Malt UF, Karlehagen S, Hoff $H$ et al. The effect of major railway accidents on the psychological health of train drivers - I. Acute psychological responses to accident. Journal of Psychosomatic Research 1993; 37: 793-805

$18 \mathrm{Yum}$ BS, Roh JH, Ryu JC et al. Symptoms of PTSD according to individual and work environment characteristics of Korean railroad drivers with experience of person-under-train accidents. Journal of Psychosomatic Research 2006; 61: 691-697

19 Mehnert A, Nanninga I, Fauth $M$ et al. Course and predictors of posttraumatic stress among male train drivers after the experience of 'person under the train' incidents. Journal of Psychosomatic Research 2012; 73: 191-196

20 Angenendt J, Nyberg E, Frommberger U. Posttraumatische Belastungsreaktionen bei Verkehrsunfallopfern. In: Maercker A, Hrsg. Posttraumatische Belastungsstörungen. Springer, Berlin Heidelberg: 2009; 501-515

21 Kim HR, Yim HW, Jo SJ et al. Major depressive disorder, panic disorder and post-traumatic stress disorder in Korean subway drivers. Int Arch Occup Environ Health 2013; 86: 471-477

22 Clarner A, Graessel E, Scholz J et al. Work-related post-traumatic stress disorder (PTSD) and other emotional diseases as consequence of traumatic events in public transportation. A systematic review. Int Arch Occup Environ Health 2014 [Epub ahead of print]

23 Brauchle G. Persistierende Dissoziation als Prädiktor posttraumatischer Belastungsstörungen bei psychosozialen Fachkräften. Persistent dissociation as predictor of posttraumatic stress disorder in emergency service professionals. Psychother Psychosom Med Psychol 2006; 56: 342-346

24 Neugebauer EA, Icks A, Schrappe M. Memorandum III: Methods for Health Services Research (Part 2). Gesundheitswesen 2010; 72: 739-748

25 Gräßel E, Donath C, Alfons $H$ et al. Versorgungsforschung - evidenzbasiert. Ein Kurzüberblick und Implikationen. Gesundheitswesen 2014, [Epub ahead of print] doi:10.1055/s-0034-1382042 